\title{
AVALIAÇÃO FARMACÊUTICA DA PRESCRIÇÃO DE NUTRIÇÃO PARENTERAL
}

\section{ARTIGO ORIGINAL}

SILVA, Alex Sander Lopes da ${ }^{1}$, COMARELLA, Larissa²

SILVA, Alex Sander Lopes da. COMARELLA, Larissa. Avaliação farmacêutica da prescrição de nutrição parenteral. Revista Científica Multidisciplinar Núcleo do Conhecimento. Ano. 06, Ed. 09, Vol. 01, pp. 26-40. Setembro 2021. ISSN: 24480959, Link de acesso: https://www.nucleodoconhecimento.com.br/saude/nutricaoparenteral, DOI: 10.32749/nucleodoconhecimento.com.br/saude/nutricao-parenteral

\section{RESUMO}

A Terapia de Nutrição Parenteral (TNP) é o método de aporte nutricional utilizado para pacientes clinicamente estáveis, sem condições de utilizar o trato gastrintestinal. É acompanhada por uma Equipe Multiprofissional de Terapia Nutricional (EMTN), que deve ser composta, no mínimo, por um médico, um nutricionista, um farmacêutico e um enfermeiro. Dentre as diversas responsabilidades do profissional farmacêutico da EMTN, encontra-se a avaliação farmacêutica da prescrição de nutrição parenteral (NP) quanto à sua adequação, concentração, compatibilidade físico-química e dosagem de administração. Entretanto, quais critérios, protocolos clínicos e padrões para concentração dos componentes da NP, interações físico-químicas e incompatibilidades devem fazer parte do rol de conhecimentos técnicos do farmacêutico para a produção de uma NP segura? Com o intuito de auxiliar neste desafio, o presente trabalho objetivou relatar as etapas da avaliação farmacêutica da prescrição de NP, seus principais parâmetros e pontos críticos, através de uma revisão de literatura, embasada em periódicos, livros e acesso a bancos de dados de publicações acadêmicas

\footnotetext{
${ }^{1}$ Mestrando em Ciências Farmacêuticas (PPGFARMA, UNEB); especialista em Farmácia Hospitalar (Uninter); especialista em Gestão da Qualidade e Gestão Ambiental (Uninassau); bacharel em Farmácia com habilitação em Indústria (UFVJM).

${ }^{2}$ Orientadora.
}

RC: 96233

Disponível em: https://www.nucleodoconhecimento.com.br/saude/nutricao-parenteral 
existentes na internet. A avaliação da osmolaridade, tipo de acesso, dos macronutrientes, eletrólitos, vitaminas, oligoelementos, interação cálcio versus fósforo, interação cálcio versus magnésio e número crítico de agragação (CAN) se mostraram como os desafios que necessitam de particular atenção ao se efetuar a avaliação farmacêutica da prescrição de NP.

Palavras-chave: nutrição parenteral, avaliação, prescrição, farmacêutico.

\section{INTRODUÇÃO}

Dentre as inúmeras atividades da assistência farmacêutica, encontra-se a análise da prescrição médica, atividade imprescindível ao farmacêutico e ao bom desempenho nos cuidados à saúde dos pacientes. Trata-se de uma análise minuciosa da prescrição, observando os parâmetros de indicação terapêutica, posologia dos itens prescritos, interações medicamentosas, interações alimento/medicamento, via de administração, e quando houver manipulação de fórmulas, análise dos parâmetros farmacotécnicos, físico-químicos e de compatibilidade e estabilidade (BRASIL, 1973; BRASIL, 1998).

A análise da prescrição mantem sua importância e toma nuances próprias na Terapia de Nutrição Parenteral (TNP), na qual a assistência farmacêutica se expressa através das etapas pertinentes à avaliação farmacêutica, manipulação, controle de qualidade, conservação e transporte (BRASIL, 1998).

O Ministério da Saúde define Nutrição Parenteral (NP), através da Portaria 272/1998, como sendo uma solução ou emulsão, composta basicamente de carboidratos, aminoácidos, lipídios, vitaminas e minerais, estéril e apirogênica, acondicionada em recipiente de vidro ou plástico, destinada à administração intravenosa em pacientes desnutridos ou não, em regime hospitalar, ambulatorial ou domiciliar, visando à síntese ou manutenção dos tecidos, órgãos ou sistemas (BRASIL, 1998). 
Neste contexto, a avaliação farmacêutica da nutrição parenteral é a análise da prescrição/formulação, quanto à sua concentração, compatibilidade físico-química de seus componentes e dosagem. Trata-se de uma atividade exclusiva do profissional farmacêutico, de extrema importância para a TNP e para o bom andamento dos cuidados oferecidos pela Equipe Multiprofissional aos pacientes submetidos à Terapia Nutricional Parenteral (TNP) (BRASIL, 1998).

A avaliação farmacêutica da nutrição parenteral é uma etapa muito importante da TNP, pois nela pode-se verificar e corrigir qualquer irregularidade na prescrição, impedindo a manipulação de uma NP inadequada. O profissional farmacêutico deve ter ciência dos parâmetros a ser analisados e, principalmente, das potenciais incompatibilidades físico-químicas que inviabilizam a produção de uma NP segura.

Entretanto, quais critérios, protocolos clínicos e padrões para concentração dos componentes da NP, interações físico-químicas e incompatibilidades devem fazer parte do rol de conhecimentos técnicos do farmacêutico para a produção de uma NP segura? Com o intuito de auxiliar neste desafio, o presente trabalho objetivou relatar as etapas da avaliação farmacêutica da prescrição de NP, seus principais parâmetros e pontos críticos.

\section{DESENVOLVIMENTO}

\subsection{METODOLOGIA}

O presente trabalho foi construído a partir de uma abrangente revisão da literatura, por meio de livros especializados, sites com banco de dados de produção acadêmica e científica, a exemplo do Scielo, além de uma extensa consulta a toda a legislação pertinente ao tema, como Portarias do Ministério da Saúde, Leis Federais e recomendações da ANVISA e do Conselho Federal de Farmácia (CFF). 


\subsection{RESULTADOS E DISCUSSÃO}

\subsubsection{A ATUAÇÃO DO FARMACÊUTICO NA EQUIPE MULTIPROFISSIONAL DA TNP}

A TNP é o conjunto de procedimentos terapêuticos para manutenção ou recuperação do estado nutricional de pacientes por meio de NP e somente pode ocorrer mediante acompanhamento de Equipe Multiprofissional de Terapia Nutricional (EMTN), constituída de, pelo menos um profissional médico, um farmacêutico, um enfermeiro e um nutricionista, habilitados e com treinamento específico para a prática da Terapia Nutricional (BRASIL, 1998).

A importância da EMTN é mundialmente aceita e há recomendações, tanto técnicas quanto legais, de que a Terapia Nutricional, principalmente a TNP, não ocorra sem o acompanhamento constante de todos os profissionais nela envolvidos. A padronização de protocolos clínicos pela EMTN, nas unidades que prestam TN e o acompanhamento constante dos pacientes pela EMTN são fundamentais para o sucesso da terapia, principalmente no que tange a sua segurança e devem haver protocolos específicos para pacientes neonatais, pediátricos, adultos e pacientes em situações específicas, como hepatopatas e nefropatas, considerando as peculiaridades dos organismos nas diversas etapas da vida e nas complicações clínicas que possam estar presentes (LEITE et al., 2005; GULIN; LOPEZ GARCIA, 2010; GUIMARÃES, 2011; BERMUDEZ; SANABRIA; VEIGA, 2013; PINTO; CASTRO; REIS, 2015; SCHOENENBERGER; RODRIGUEZ, 2015).

As atribuições do profissional farmacêutico na EMTN compreendem, desde a seleção, obtenção, armazenamento e distribuição de todo os produtos necessários ao preparo da NP, à supervisão nas rotinas operacionais de preparação (BRASIL, 1998).

Para tanto, também faz parte de suas responsabilidades, a qualificação dos fornecedores; o acompanhamento dos certificados e laudos de análise dos fabricantes; a avaliação da formulação da prescrição médica; o uso de técnicas pré- 
estabelecidas de preparação da NP que assegurem compatibilidade físico-química, esterilidade, apirogenicidade e ausência de partículas; a determinação do prazo de validade de cada NP padronizada, com base em critérios rígidos de controle de qualidade; a correta amostragem para análise microbiológica; a correta rotulagem do produto acabado; a análise de reações adversas e interações droga-nutrientes e nutriente-nutriente, a partir do perfil farmacoterapêutico registrado; o treinamento, a educação continuada e a atenta supervisão dos colaboradores do setor de manipulação / farmácia, além da manutenção dos registros legais obrigatórios, como data e hora de preparação da NP, nome completo do paciente, identificação (nome e registro) do médico e do manipulador (BRASIL, 1998).

\subsubsection{VISÃO GERAL DA ANÁLISE DA PRESCRIÇÃO DA NUTRIÇÃO PARENTERAL}

A Avaliação Farmacêutica da prescrição de NP é atividade obrigatória, segundo a Legislação Brasileira e especificada na Portaria 272/1998 do Ministério da Saúde:

A avaliação farmacêutica da prescrição da NP quanto à sua adequação, concentração e compatibilidade físico-química de seus componentes e dosagem de administração, deve ser realizada pelo farmacêutico antes do início da manipulação. Qualquer alteração na prescrição, que se fizer necessária, em função da avaliação farmacêutica, deve ser discutida com o médico da equipe que é o responsável por sua alteração formal (BRASIL, 1998).

Nesse contexto, cabe ao profissional farmacêutico avaliar a prescrição item por item, além da estabilidade físico-química da mistura resultante, discutir com o profissional médico as adequações que porventura se fizerem necessárias e, caso existam, a partir do acordo resultante, solicitar uma nova prescrição com as alterações efetuadas (BRASIL, 1998).

É muito importante que essa etapa seja efetuada com total cuidado e interação entre a EMTN. É de conhecimento empírico e também demonstrado por estudos (GUIMARÃES, 2011) que há inconformidades na grande maioria das prescrições de NP e é na Avaliação Farmacêutica da Prescrição que essas inconformidades podem ser observadas e corrigidas antes da manipulação da NP. 
A primeira etapa para a prescrição da NP é a avaliação do estado nutricional do paciente e de suas funções clínicas vitais, pois a NP somente deve ser iniciada em pacientes que estejam com as funções vitais estáveis e com previsão mínima de tratamento por três dias para pacientes desnutridos e de cinco dias para pacientes eutróficos, impossibilitados de utilizar o trato gastrointestinal, para justificar o risco/benefício do tratamento (FERRERIA, 2007). Além disso, é importante que a NP seja iniciada com $30 \%$ a $50 \%$ das necessidades nutricionais estimadas, progredindo gradualmente para $100 \%$ entre 48 a 72 horas do início da terapia, a fim de reduzir os riscos de distúrbios metabólicos, principalmente da síndrome de realimentação, principalmente nos indivíduos hipermetabólicos com inanição grave (CALIXTO-LIMA et al., 2011, p.35). Essa etapa deve ser efetuada pelo nutricionista e pelo médico nutrólogo da EMTN e revisada pelo farmacêutico, na avaliação da prescrição.

\subsubsection{AVALIAÇÃO DA OSMOLARIDADE E ADEQUAÇÃO AO TIPO DE ACESSO}

A Osmolaridade é a concentração de partículas ativas na solução, ou seja, de partículas capazes de exercer pressão osmótica, por litro de solução (BAXTER; WAITZBERG; RODRIGUES, 2000, p. 659). Há diversas fórmulas para se calcular a osmolaridade final da NP, porém, o método mais preciso consiste em verificar a informação fornecida por cada fabricante para cada um dos componentes a ser inseridos na formulação e depois somar a contribuição individual de cada um deles, pois a osmolaridade varia em função das diferenças, mesmo que mínimas, nos componentes dos insumos (CALIXTO-LIMA et al., 2011, p.58).

Não há um consenso sobre qual é a osmolaridade ideal máxima para infusão de NP via acesso periférico (veias de pequeno calibre, geralmente no braço). Alguns autores sugerem $600 \mathrm{mOsmol} / \mathrm{L}$ (SOUZA, 2008, p.278-89), enquanto outros consideram aceitável até $900 \mathrm{mOsmol} / \mathrm{L}$ para infusão de NP em acesso periférico (COSTA, 2014, p.38).

Na prática clínica, a Equipe Multiprofissional define o padrão para a instituição que representam, dentro dos limites cientificamente estabelecidos. Definido o ponto de 
corte, entre 600 a $900 \mathrm{mOsmol} / \mathrm{L}$, esse passa a ser o padrão institucional para a definição do tipo de acesso aceitável para a NP. Cabe ao profissional farmacêutico calcular precisamente a osmolaridade final da solução da NP e, caso a osmolaridade seja superior à definida para acesso periférico, discutir com a EMTN a troca do acesso do paciente para o tipo central (cateter inserido em veia de grande calibre, geralmente a veia cava superior) ou, caso não seja viável, solicitar ao médico prescritor a adequação da prescrição para que a osmolaridade se enquadre para acesso periférico, sugerindo ajustes mínimos que o permitam, como uma diminuição pequena de no aporte de glicose e aminoácidos, por exemplo.

\subsubsection{AVALIAÇÃO DOS MACRONUTRIENTES}

São considerados macronutrientes na NP os aminoácidos, a glicose e a emulsão lipídica, quando presente. São esses os elementos que contribuirão para o aporte energético do paciente (CALIXTO-LIMA et al., 2011, p.58). O cálculo do Valor Energético Total (VET) é efetuado pelo nutricionista ou pelo médico nutrólogo da EMTN e esse aporte calórico é dividido na prescrição através da oferta dos macronutrientes. Caso o paciente necessite de ganho de peso / massa corporal, o médico prescritor determinará um aporte calórico na NP superior ao VET, fazendo com que a oferta calórica seja maior do que o gasto energético.

O Quadro 1 demonstra a recomendação da prescrição de macronutrientes para pacientes adultos em TNP. É importante frisar que os valores dependem da tolerância individual de cada paciente e valores inferiores ou superiores não representam erro de prescrição, desde que ocorram mediante justificativa clínica plausível.

Quadro 1: Prescrição Recomendada para pacientes adultos em Nutrição Parenteral

\begin{tabular}{|l|l|l|}
\hline MACRONUTRIENTES & PACIENTES GRAVES & PACIENTES ESTÁVEIS \\
\hline PROTEÍNAS & 1,2 A $1,5 \mathrm{~g} / \mathrm{Kg} / \mathrm{dia}$ & $0,8 \mathrm{a} 1 \mathrm{~g} / \mathrm{Kg} / \mathrm{dia}$ \\
\hline
\end{tabular}

RC: 96233

Disponível em: https://www.nucleodoconhecimento.com.br/saude/nutricao-parenteral 


\begin{tabular}{|l|l|l|}
\hline CARBOIDRATOS & $\leq 3 \mathrm{mg} / \mathrm{Kg} / \mathrm{min}$ & $\leq 5 \mathrm{mg} / \mathrm{Kg} / \mathrm{min}$ \\
\hline LIPÍDEOS & $1 \mathrm{~g} / \mathrm{Kg} / \mathrm{dia}$ & 1 a $2 \mathrm{~g} / \mathrm{Kg} / \mathrm{dia}$ \\
\hline
\end{tabular}

Fonte: Calixto-Lima et al., 2011, p.36

Existem no mercado soluções especiais de aminoácidos para pacientes nefropatas, apenas com aminoácidos essenciais e histidina, soluções especiais para pacientes hepatopatas, com diminuição no percentual de aminoácidos aromáticos e soluções especiais de aminoácidos para pacientes neonatais e pediátricos, com perfil do leite materno. É importante que a solução de aminoácidos na NP corresponda à necessidade clínica do paciente e que as soluções especializadas para nefropatas, hepatopatas e as soluções pediátricas sejam utilizadas quando este for o perfil do paciente, para garantir uma maior segurança e eficácia na terapia (CALIXTO-LIMA et al., 2011, p.6).

A glutamina, aminoácido mais abundante no plasma e substrato principal das células imunes e enterócitos, é um aminoácido considerado condicionalmente essencial em processos catabólicos e pode ser ministrado em soluções dipeptídicas físicoquimicamente estáveis (FERREIRA, 2007; CALIXTO-LIMA, 2011). Não há evidencias científicas suficientes para o uso rotineiro de glutamina na NP, entretanto, pacientes com queimaduras e traumas são amplamente beneficiados com seu uso, havendo menor índice de complicações infecciosas e uma redução significativa no tempo de internamento, nesses casos (FERREIRA, 2007).

\subsubsection{AVALIAÇÃO DOS ELETRÓLITOS}

Os eletrólitos são essenciais para manutenção do equilíbrio osmótico, do equilíbrio ácido-base, bem como das mais variadas funções celulares e devem estar presentes, em doses terapêuticas, diariamente na NP, a fim de manter a homeostase. 
As necessidades de cada paciente para cada um dos eletrólitos disponibilizados devem ser cuidadosamente avaliadas mediante seu histórico clínico. Devem-se levar em consideração as comorbidades, o estado das funções hepáticas e renais, a interferência dos medicamentos utilizados pelo paciente na concentração plasmática dos eletrólitos (a hidroclorotiazida provoca maior depleção de potássio, por exemplo), as condições cardíacas e respiratórias, bem como as perdas extrarrenais, como diarreia e emese (CALIXTO-LIMA et al., 2011, p.22).

Há padrões para determinação da prescrição de eletrólitos individualizados para pacientes adultos, pediátricos e neonatais, cada um com seus respectivos limites de recomendação diária. O padrão de eletrólitos para pacientes adultos, sem disfunção orgânica associada e sem perdas consideráveis de eletrólitos por vias extrarrenais é apresentado no Quadro 2.

Quadro 2: Recomendações Diárias de Eletrólitos

\section{ELETRÓLITO RECOMENDAÇÃO / DIA}

\begin{tabular}{|l|l|}
\hline Cálcio & 10 a $15 \mathrm{mEq}$ \\
\hline Magnésio & 8 a $20 \mathrm{mEq}$ \\
\hline Fosfato & 20 a $40 \mathrm{mMol}$ \\
\hline Sódio & 1 a $2 \mathrm{mEq} / \mathrm{Kg}+$ reposição \\
\hline Potássio & 1 a $2 \mathrm{mEq} / \mathrm{Kg}$ \\
\hline Acetato & conforme necessário para manter o balanço ácido básico \\
\hline Cloreto & conforme necessário para manter o balanço ácido básico \\
\hline
\end{tabular}

Fonte: Calixto-Lima et al., 2011, p.23

É importante ressaltar que os limites posológicos dos padrões de prescrição podem e devem ser ultrapassados, se houver necessidade clínica sinalizada pelo médico prescritor, como hipocalemia severa, dentre outros. Cabe ao farmacêutico avaliar se as recomendações posológicas estão sendo seguidas, ou, caso não estejam, verificar com o médico prescritor a real presença de necessidade clínica para doses 
não-padronizadas a fim de garantir a segurança do paciente em uso de TNP (BRASIL, 1998).

O Ferro não é adicionado a NP e deve ser administrado separadamente por via venosa, devido a sua alta reatividade química com os demais componentes da formulação, que pode propiciar a formação de quelatos, além de provocar instabilidade na emulsão lipídica (FERREIRA, 2007).

\subsubsection{AVALIAÇÃO DOS OLIGOELEMENTOS E DAS VITAMINAS}

Oligoelementos ou elementos-traço são minerais que atuam como co-fatores enzimáticos. Sem estar associadas a esses micronutrientes, muitas enzimas perdem sua atuação como catalizadora dos processos bioquímicos e todo o funcionamento do organismo fica comprometido. Estes minerais devem ser adicionados à NP em doses e apresentações que não alterem a estabilidade físico-química da solução (CALIXTO-LIMA, 2011, p.26). Também devem-se monitorá-los constantemente, principalmente com relação ao fósforo, ao magnésio e ao zinco para, se necessário, adequar a oferta a fim de manter níveis séricos normais (FERREIRA, 2007). A recomendação diária de aporte de oligoelementos na NP para pacientes adultos é apresentada no Quadro 3.

Quadro 3: Recomendação de Elementos-Traço para Pacientes Adultos em Nutrição Parenteral

\begin{tabular}{|l|l|}
\hline $\begin{array}{l}\text { ELEMENTO- } \\
\text { TRAÇO }\end{array}$ & RECOMENDAÇÃO / DIA \\
\hline Zinco & 2,5 a $5 \mathrm{mg} / \mathrm{dia}$ \\
\hline Cobre & 0,3 a $0,5 \mathrm{mg} / \mathrm{dia}$ \\
\hline Cromo & 10 a $15 \mu \mathrm{g} / \mathrm{dia}$ \\
\hline Manganes & 60 a $100 \mu \mathrm{g} / \mathrm{dia}$ \\
\hline
\end{tabular}

Fonte: American Society for Parenteral and Enteral Nutrition, 1998, por Calixto-Lima et al., 2011, p.26 
Vitaminas são substâncias essenciais para o metabolismo e que o organismo não consegue sintetizar, devendo, portanto, obtê-las da dieta. Não estão bem estabelecidas as necessidades de vitaminas nos pacientes críticos (FERREIRA, 2007), porém, há recomendações diárias para pacientes adultos (MARCHINI, 1998) pode ser visualizada no Quadro 4.

Quadro 4: Doses Recomendadas de Vitaminas em pacientes adultos

\begin{tabular}{|l|l|}
\hline VITAMINA & DOSE/DIA \\
\hline Vitamina A & $1.000 \mu \mathrm{g}$ \\
\hline Vitamina B1 & $3 \mathrm{mg}$ \\
\hline Vitamina & $5 \mu \mathrm{g}$ \\
B12 & \\
\hline Vitamina B2 & $3,6 \mathrm{mg}$ \\
\hline Vitamina B6 & $4 \mathrm{mg}$ \\
\hline Vitamina C & $100 \mathrm{mg}$ \\
\hline Vitamina D & $5 \mathrm{a} 10 \mu \mathrm{g}$ \\
\hline Vitamina E & $10 \mathrm{a} 15 \mathrm{mg}$ \\
\hline Vitamina K & $200 \mu \mathrm{g}$ \\
\hline
\end{tabular}

Fonte: Adaptado de Marchini, 1998.

É importante ressaltar que a Vitamina $\mathrm{K}$ não está presente nas multivitaminas que temos no mercado para uso em NP, pois pode causar sérias alterações em pacientes em uso de anticoagulantes. Além disso, a Vitamina $\mathrm{K}$ costuma inativar a Vitamina C, assim como o faz a Vitamina B12 (SPOLIDORO, 2000). Caso seja necessária, a Vitamina $\mathrm{K}$ deve ser fornecida por outro meio (FERREIRA, 2007).

Caso seja padrão da instituição manipuladora produzir a NP em duas bolsas, separando a bolsa da emulsão lipídica dos demais componentes, sugere-se separar também os oligoelementos das vitaminas, pois o cobre e o zinco podem degradar a Vitamina C. Essa reação pode ser visualmente percebida, caso ocorra, por uma coloração azulada na NP (SPOLIDORO, 2000). 
Devido à inatividade da Vitamina B12 na NP, em pacientes em TNP prolongada, aconselha-se a administração de Vitamina B12 via intramuscular $100 \mathrm{mg} / \mathrm{mês}$ ou $300 \mathrm{mg}$ a cada 3 meses (SPOLIDORO, 2000).

\subsubsection{AVALIAÇÃO DA RELAÇÃO CÁLCIO X FÓSFORO}

É sabido que íons cálcio e íons fosfato, a partir de determinada concentração crítica em solução, tendem a se agrupar formando fosfato de cálcio, um precipitado insolúvel. Isso acontece principalmente quando o fósforo é fornecido como fosfato de potássio, o que deixa o fosfato livre para a ocorrência da reação química de precipitação. É considerada uma relação ideal a de $1,7 \mathrm{mg}$ de cálcio para cada miligrama de potássio, sendo considerada máxima a soma das concentrações de Cálcio e Fósforo em 40mEq/L ou uma relação de Cálcio x Fósforo igual a 250mEq/L. Valores maiores são altamente indicativo de ocorrência de instabilidade físicoquímica por formação de precipitado de fosfato de cálcio, inviabilizando a manipulação da prescrição por instabilidade físico-química da solução resultante. $\mathrm{O}$ fornecimento do potássio na forma de glicerofosfato de potássio não deixa os íons fosfato livres para que ocorra a reação de precipitação com o cálcio e dispensa os cálculos de estabilidade físico-química com relação a esse parâmetro (SPOLIDORO, 2000).

\subsubsection{AVALIAÇÃO DA RELAÇÃO CÁLCIO X MAGNÉSIO}

A relação entre íons Cálcio e íons Magnésio também é um fator a ser monitorado para garantir a estabilidade da solução da NP. É sabido que uma relação Cálcio + Magnésio superior a $16 \mathrm{mEq} / \mathrm{L}$ tende a desestabilizar a emulsão lipídica por pressão iônica ou neutralização das cargas dos emulsificantes dos lipídeos, forçando a abertura e agregação das micelas, com consequente separação da fase oleosa da fase aquosa da solução (Souza, 2008; SPOLIDORO 2000). 


\subsubsection{AVALIAÇÃO DO NÚMERO CRÍTICO DE AGREGAÇÃO (CAN)}

O Número Crítico de Agregação, também conhecido como CAN (Critical Number Aggregation) é um dos parâmetros que avaliam a possibilidade de separação de fases ou ruptura da solução de NP. É calculado pela fórmula de Shultz-Hard:

$\mathrm{CAN}=(\mathrm{A}+64 \times \mathrm{B}+729 \times \mathrm{C}) \times 1000 /$ Volume total da NP, onde: $\mathrm{A}=$ concentração de cátions monovalentes em $\mathrm{mMol} / \mathrm{L}$

$B=$ concentração de cátion bivalentes em $\mathrm{mMol} / \mathrm{L} C=$ concentração de cátions trivalentes em $\mathrm{mMol} / \mathrm{L}$

Neste cálculo, todos os íons que podem neutralizar as cargas dos emulsificantes presentes na emulsão lipídica, promovendo aumento no tamanho das micelas, ou mesmo separação total das fases aquosa e oleosa são considerados, de acordo com sua valência química para tal, sendo recomendado um valor entre 400 a 700 $\mathrm{mMol} / \mathrm{L}$ (GASTALDI, 2009). Valores de CAN superiores a 700 são impeditivos da manipulação, devendo o médico prescritor ser comunicado do fato e a prescrição ser adequada ao parâmetro com redução de íons, principalmente os divalentes, como cálcio e magnésio, a fim de promover uma produção de NP segura (GASTALDI, 2009).

\section{CONSIDERAÇÕES FINAIS}

A Avaliação Farmacêutica da Prescrição de Nutrição Parenteral é uma etapa crítica da TNP, é obrigatória segundo a Legislação Brasileira e recomendada por todas as grandes instituições mundiais ligadas à saúde.

Cabe ao profissional farmacêutico, como membro ativo da EMTN fazer uma avaliação rigorosa da prescrição médica, impedindo a manipulação de NP's inadequadas, com relação à concentração, compatibilidade físico-química de seus componentes e dosagem de administração, sinalizando e discutindo com o médico prescritor as alterações necessárias para a produção de uma NP segura e eficaz 
para o paciente em TNP, obtendo do mesmo uma prescrição adequada à terapia, antes de iniciar a manipulação.

A criação e padronização de protocolos nas unidades que prestam serviço de TNP, seguindo as recomendações científicas conhecidas, uma boa interação entre os profissionais da EMTN, treinamento e aperfeiçoamento constantes, são os principais meios para aumentar a eficácia e, principalmente, a segurança da Terapia Nutricional como um todo, incluindo a Avaliação Farmacêutica da Prescrição da NP.

\section{REFERÊNCIAS}

BRASIL. Lei $n^{\circ} 5.991$ de 17 de Dezembro de 1973. Dispõe sobre o Controle Sanitário do Comércio de Drogas, Medicamentos, Insumos Farmacêuticos e Correlatos, e dá outras providências. Diário Oficial da República Federativa do Brasil. Disponível em: <http://www.cff.org.br/userfiles/file/educacao_farmaceutica/Comissao_Ensino/Outras \%20Legislacoes/Lein5991_1973.pdf> . Acesso em: 01/02/2021.

BRASIL - Ministério da Saúde. Portaria n²72 de 8 de Abril de 1998. Dispõe sobre os requisitos mínimos para a Terapia de Nutrição Parenteral. Diário Oficial da República Federativa do Brasil. Disponível em: http://bvsms.saude.gov.br/bvs/saudelegis/svs1/1998/prt0272_08_04_1998.html>. Acesso em: 01/02/2021.

BAXTER, YC, WAITZBERG, DL, RODRIGUES, JJG, et al. Critérios de decisão na seleção de dietas enterais. In: Waitzberg DL, editor. Nutrição oral, enteral e parenteral na prática clínica. 3a ed. São Paulo: Atheneu; 2000. p. 659-76.

BERMUDEZ, Charles; SANABRIA, Álvaro; VEGA, Valentín. Soporte nutricional del paciente adulto. Resultados de la reunión de consenso de la Asociación Colombiana de Nutrición Clínica. rev. colomb. cir., Bogotá , v. 28, n. 2, June 2013. Disponível em: <https://pesquisa.bvsalud.org/portal/resource/pt/lil-680513>. Acesso em: 01/02/2021. 
CALIXTO-LIMA, Larissa et. al. Componentes e Cálculo da Nutrição Parenteral. Editora Rubio, Rio de Janeiro RJ, 2011.

COSTA, Maria José de Carvalho; COSTA, Eliseuda Marinho da Silva. Nutrição parenteral: uma abordagem metabólica para nutricionistas. Editora da UFPB, João Pessoa, 2014. Disponível em: <http://www.editora.ufpb.br/sistema/press5/index.php/UFPB/catalog/download/203/7 0/752-1 ?inline=1 >. Acesso em: 01/02/2021.

FERREIRA, lára Kallyanna Cavalcante. Terapia Nutricional em Unidade de Terapia Intensiva. Revista Brasileira de Terapia Intensiva Vol. 19 № 1, São Paulo, Janeiro - Março, 2007. Disponível em: <https://www.scielo.br/scielo.php?pid=S0103507X2007000100012\&script=sci_abstract\&tlng=pt>. Acesso em: 01/02/2021.

GASTALDI, Marcelo. Farmácia Hospitalar - Nutrição Parenteral Total: Da Produção a Administração. Encarte Pharmacia Brasileira, set-out. 2009. Disponível em: https://www.cff.org.br/sistemas/geral/revista/pdf/122/encarte_farmAcia_hospitalar_pb 72.pdf>. Acessoa em: 01/02/2021.

GUIMARAES, Daniel Rossi de Santiago; et. al. Avaliação das Prescrições de Nutrição Parenteral dos Usuários de um Hospital Público de Fortaleza. Rev. Bras. Farm. Hosp. Serv. Saúde. São Paulo, v. 3, n. 2, p. 25-29, Abr./Jun. 2012. Disponível em: <http://www.v1.sbrafh.org.br/public/artigos/2012030205BR.pdf>. Acessado em: 01/02/2021.

GULIN DAVILA, J.; LOPEZ GARCIA, V. M.. Aplicación de estándares a la monitorización del soporte nutricional parenteral en el paciente adulto. Nutr. Hosp., Madrid, $\quad$ v. $25, \quad$ n. 3, jun. 2010. Disponível em

$<$ http://scielo.isciii.es/scielo.php?script=sci_arttext\&pid=S0212-16112010000300016 >. Acesso em: 01/02/ 2021.

LEITE, Heitor Pons; CARVALHO, Werther Brunow de; SANTANA E MENESES, Juliana Fernandez. Atuação da equipe multidisciplinar na terapia nutricional de 
pacientes sob cuidados intensivos. Rev. Nutr., Campinas, v. 18, n. 6, p. 777-784, Dec. 2005.

em:

<http://www.scielo.br/scielo.php?script=sci_arttext\&pid=S1415-

$52732005000600008 \& \operatorname{lng}=e n \& n r m=i s o>$. Acesso em: 05/02/2021.

MARCHINI, J.S. et al. Nutrição Parenteral - Princípios Gerai, Formulários de Prescrição e Monitorização. Medicina, Ribeirão Preto, 31: 62-72, jan./mar. 1998. Disponível em: <https://www.revistas.usp.br/rmrp/article/view/7635df>. Acesso em: 05/02/2021.

PINTO, Isabela Vaz Leite; CASTRO, Mariza dos Santos; REIS, Adriano Max Moreira. Descrição da Atuação do Farmacêutico em Equipe Multiprofissional com Ênfase no Cuidado ao Idoso Hospitalizado. Rev. bras. geriatr. gerontol., Rio de Janeiro , v. 16, n. 4, p. 747-758, Dec. 2013 . Disponível em:<https://www.scielo.br/scielo.php?pid=S180998232013000400747\&script=sci_abstract\&tIng=pt>. Acesso em: 05/02/2021.

SCHOENENBERGER ARNAIZ, J. A.; RODRIGUEZ POZO, A.. Protocolización de la Nutrición Artificial por vía Parenteral: Bases metodológicas y organizativas para el diseño y revisión del proceso. Nutr. Hosp., Madrid, v. 25, n. 1, feb. 2010 . Disponível em: $<$ http://scielo.isciii.es/scielo.php?script=sci_arttext\&pid=S021216112010000100004>. Acesso em: 05/02/2021.

SOUZA, Fabíola Isabel $\mathrm{S}$. de et al. Nutrição Parenteral no rescém-nascido prétermo: proposta de protocolo prático. Ver. Paul. Pediatr., São Paulo, 2008; 26(3):278-89. Disponível em < https://www.scielo.br/scielo.php?pid=S010305822008000300013\&script=sci_abstract\&tIng=pt>. Acesso em: 05/02/2021.

SPOLIDORO, José Vicente Noronha. Nutrição parenteral em Pediatria. J. pediatr., Rio de Janeiro, V.76,. p. 339-348, 2000. Disponível em: < http://www.jped.com.br/ArtigoDetalhe.aspx?varArtigo=173\&idioma=pt-BR $>$. Acesso em:05/02/2021. 
Enviado: Março, 2021.

Aprovado: Setembro, 2021.

RC: 96233

Disponível em: https://www.nucleodoconhecimento.com.br/saude/nutricao-parenteral 